\title{
LA INICIATIVA DEL CINTURÓN Y LA \\ RUTA DE LA SEDA (BRI): GLOBALIZACIÓN INCLUYENTE COMO MOTOR PARA \\ LA TRANSICIÓN PACÍfICA DE CHINA AL LIDERAZGO MUNDIAL
}

Juan Camilo Vejarano Rivera ${ }^{(a)}$

THE BELT AND SILK ROAD INITIAVE: INCLUSIVE

GLOBALIZATION AS THE DRIVER OF CHINA'S PEACEFUL

TRANSITION TOWARDS GLOBAL LEADERSHIP

A INICIATIVA DO CINTURÃO E A ROTA DA SEDA:

GLOBALIZAÇÃO INCLUSIVA COMO MOTOR PARA A TRANSIÇÃO

PACÍFICA DE CHINA À LIDERANÇA MUNDIAL

Fecha de recepción: 5 de abril del 2019

Fecha de aprobación: 19 de junio del 2019

Disponible en línea: 1 de julio del 2019

Sugerencia de citación:

Vejarano Rivera, J.C. (2019). La iniciativa del cinturón y la ruta de la seda (BRI): globalización incluyente como motor para la transición pacífica de China al liderazgo mundial. Razón Crítica, 7, 111-138, doi: $10.21789 / 25007807.1509$

(a) Juan Camilo Vejarano Rivera

Profesional en Gobierno y Relaciones Internacionales de la Universidad Externado de Colombia, Colombia

https://orcid.org/0000-0002-0761-9004

jcvejaranorivera@gmail.com 


\section{R E S U M E N}

La importancia de la cultura china como civilización y como estado se remonta a 5.000 años de historia documentada, en la cual sucesivas generaciones mantienen el conocimiento, la técnica y el comercio, como tradición y legado de su cultura. A través de los siglos, China se ha transformado como estado y también ha modificado las maneras de relacionarse con el resto del mundo, es decir, desde la ruta de la seda, hasta los nuevos modelos comerciales. En la actualidad, el mundo globalizado ha impuesto sobre la tradición china un nuevo reto, cómo insertarse en el contexto mundial luego de presentar un crecimiento y desarrollo económico a tasas elevada, hasta el pasado año, el 2018, cuando la tasa de crecimiento bajó, pero la presencia de China en el mundo aumentó sustancialmente. Se presenta a China como una civilización, que a partir del discurso ha construido la estrategia con que buscará el liderazgo mundial, basado en el apoyo al mundo en desarrollo. Lo anterior, a la luz de las relaciones internacionales y los planteamientos de la teoría de los complejos regionales de seguridad, este análisis cobra pertinencia y relevancia al observarse los cambios que esto podría generar en los límites de los complejos del mundo posterior a la guerra fría.

\section{PALABRAS CLAVE: China, relaciones internacionales, Ruta de la Seda, securitization ${ }^{1}$ (segurizar-segurización), globalización incluyente, liderazgo mundial, Belt Road Initiative- La Iniciativa del Cinturón y la Ruta de la Seda).}

1. Securitization: el pronunciamiento de "seguridad" se puede ver como un acto por el cual se pueden organizar todo tipo de problemas (militares, políticos, económicos, ambientales, etc.) como una amenaza. Un discurso en términos de "securitization" debe seguir una estructura retórica específica, derivada de la guerra y sus connotaciones históricas de supervivencia, urgencia, amenaza y defensa. La Escuela de Copenhague define "securitization" como un acto de construcción discursiva ejecutado por un actor/sujeto que debe cumplir tres criterios retóricos:

(1) Afirma que un objeto referente está existencialmente amenazado;

(2) Exige el derecho de tomar contramedidas extraordinarias para hacer frente a esa amenaza:

(3) Convence a una audiencia que gobierna y justifica romper el comportamiento regular para contrarrestar la amenaza.

De acuerdo a lo estipulado anteriormente, etiquetar algo como seguro es un problema que se eleva como un asunto de prioridad suprema (Van Muester, 2012).

Securización/securitización/segurización/segurizar: el verbo segurizar-preferible a securizar, formado a partir de voces inglesas como secure o security- se encuentra ya en el Diccionario de uso del español con el significado concreto de "hacer que un cristal sea de seguridad" ("vidrio segurizado'), lo que hace posible ampliar este significado para aplicarlo a una tarjeta de crédito, al pago realizado con esta, a un ordenador o a un entorno informático ("Segurizar mejor que securizar", s. f.).

Los investigadores Claudia Sisco Marcano y Oláguer Chacón Maldonado usan la palabra "seguritización" como un proceso mediante el cual se pretende darle calidad o estatus de asunto de seguridad, esto es, a un problema que puede atentar contra la supervivencia de individuos, conglomerados, estados o la humanidad. Este término teórico proviene del inglés "securitization" (Sisco \& Chacón, 2004)

De acuerdo con la Fundéu BBVA y la RAE, se utilizará la palabra segurización y el verbo segurizar, pues securitization es un neologismo utilizado en los estudios de seguridad que no tiene traducción oficial al español. 


\section{A B S T R A C T}

The importance of the Chinese culture, as a civilization and State, goes back to 5,000 years of documented history, where successive generations have preserved knowledge, techniques and trade patterns as a tradition and a cultural legacy. Over the centuries, China has transformed as a State and modified its ways of engaging with the rest of the world, from the Silk Road to the new commercial models. These days, globalization has established a new challenge for the Chinese tradition: How to fit within the world context after a steady and fast-paced economic growth (since the 1990's) that slowed down in 2018 -although Chinese influence around the world became bigger. In the light of international relations, this analysis becomes appropriate and relevant.

KEY WORDS: China, international relations, Silk Road, securitization, inclusive globalization, global leadership.

\section{R E S U M O}

A importância da cultura chinesa, como civilização e como Estado, remonta-se a 5.000 anos de história documentada, na qual sucessivas gerações mantêm o conhecimento, a técnica e o comércio, como tradição e legado de sua cultura. A través dos séculos, China tem se transformado como Estado, e também tem mudado as maneiras de se relacionar com o resto do mundo. Isto é, desde a rota da seda até os novos modelos comerciais. Na atualidade, o mundo globalizado tem imposto à tradição chinesa um novo desafio: como se inserir no contexto mundial, depois de apresentar um crescimento e desenvolvimento econômico a taxas elevadas. Este crescimento tem se mantido de maneira constante desde os anos 1990 até o 2018, no qual a taxa de crescimento baixou, mas a presença de China no mundo aumentou substantivamente. À luz das relações internacionais e os enfoques da teoria dos complexos regionais de segurança, esta análise cobra pertinência e relevância.

PALAVRAS-CHAVE: China, relações internacionais, rota da seda, titularização, globalização inclusiva, liderança mundial. 


\section{Introducción y apuntes metodológicos}

Para la realización de este artículo fue necesaria la utilización de diferentes fuentes documentales relacionadas con la "Belt and Road Initiative" (BRI por su sigla en inglés) y China. La búsqueda comprendió la revisión, la lectura y el análisis de libros, documentos académicos, artículos de prensa, publicaciones periódicas y de algunos documentos institucionales oficiales, lo que nos lleva a definir este artículo como fruto de una investigación documental, que permite contrastar la realidad con la teoría, para explicar los diferentes efectos y fenómenos derivados de una acción o decisión de política.

En segundo lugar, fue necesario relacionar los hallazgos a la luz de teorías de las relaciones internacionales, para poder establecer el estatus actual de China en el sistema internacional y definir el impacto que tendrá la iniciativa en las reacciones de amistad y enemistad que se construyen en el sistema. En este sentido, los fundamentos teóricos estuvieron basados principalmente en el planteamiento de los complejos regionales de seguridad de Buzan, pero sin dejar de lado los postulados realistas de caracterización de las grandes potencias y el globalismo en términos de globalización incluyente para explicar los cambios que BRI puede generar en las relaciones de los sujetos internacionales.

A partir de esto, se comienza con algunos apuntes históricos que centran al lector en la trascendencia de la Belt Road Initiative, no como un capricho del autor, sino como un concepto que refleja la tradición histórica del comercio y las rutas comerciales de China en la región de influencia y ahora en el mundo globalizado.

A finales del siglo xıx, el geógrafo alemán Ferdinand von Richthofen (1833-1905) bautizó con el nombre de "ruta de la seda" a la red de comunicaciones que enlazaba a China con Occidente. Esta expresión 
resume toda una historia de intercambios entre el Este de Asia y Europa, a partir del siglo i a. C., cuando los romanos descubrieron que existía la seda en China. La Ruta de la Seda fue tanto un itinerario mercantil para el comercio de las especias, del papel y de la porcelana como una vía de intercambios intelectuales, religiosos y técnicos (Llastoguera, 2004).

Por otro lado, el sinólogo francés Edouard Chavannes demostró a principios del siglo xx que la Ruta de la Seda no comprendía únicamente los caminos terrestres que permitían los intercambios comerciales y culturales en Eurasia, sino que también comprendía históricamente las rutas marítimas comerciales existentes.

El comercio transfronterizo ha existido durante miles de años. Estudios arqueológicos e históricos muestran que el comercio entre China, Asia Central y Occidental se puede rastrear al menos 2.500 años antes del periodo de los reinos combatientes (475 a. C.-221 a. C.). Después la dinastía Qing (221 a. C.-207 a. C.), reunificó a los estados en guerra en un imperio; este comercio fue documentado con registros regulares y oficiales, mientras que los registros de la dinastía Han (202 a. C.-220 d. C.) notaron que Qian Zhang hizo varias misiones diplomáticas al oeste. Aunque su primera embajada no logró establecer una alianza con los Darouzhi (conducido más hacia el oeste hacia los tramos superiores de Amu Darya, en el norte de Afganistán para cuando la embajada llegó allí), sus reportes proporcionaron a la corte imperial con información confiable sobre posibles socios comerciales. El comercio entre China y Occidente fue posteriormente impulsado por la participación oficial del estado (Cai, 2017).

A lo largo de 2.000 años, desde las dinastías Han a Tang, Song, Yuan, Ming y Qing, el comercio continuó. Aunque prosperó y luego menguó, su alcance espacial se extendió, cubriendo la mayoría de países euroasiáticos e incluso algunas zonas de África. Aunque la gama de productos comercializados aumentó, varios fueron simbólicos: la seda, especialmente hasta que comenzó la producción doméstica (con gusanos de seda robados) en el Imperio Bizantino bajo el emperador Justiniano I; China, desde la dinastía Song, y el té en los últimos siglos. Los intercambios culturales también se producen a menudo entre socios comerciales. Un ejemplo de esto se dio durante la dinastía Han: el budismo se extendió a China, Corea y Japón a lo largo de las rutas comerciales (Cai, 2017). Otro ejemplo de esto fue la expansión del islam, debido a que el comercio marítimo en el Océano Índico y las rutas terrestres desde el medio oriente 
permitieron que se multiplicara dicha creencia por África del Norte, Asia Central y el Sudeste Asiático

\title{
China: su retórica sobre el estatus internacional y la búsqueda de este
}

\begin{abstract}
Desde la victoria de Mao Zedong, China ha buscado aumentar su estatus internacional, teniendo en cuenta que este es entendido por las élites del Partido Comunista como la obtención de poder convencional en lo militar y económico, así como el éxito de su política exterior. En este sentido, se ha propendido por la construcción de un sentimiento nacionalista, que eleve su estatus interno hacia el exterior, que, a través de la educación desde los primeros niveles, se inculque a la población el conocimiento de su cultura, los 5.000 años de historia como civilización, sus importantes contribuciones a la humanidad, el orgullo y compromiso con su tradición.
\end{abstract} Esta es la manera en que el Partido planea devolver la gloria pasada que tuvo el imperio durante los años de la Ruta de la Seda.

De acuerdo con lo anterior, durante la etapa de liderazgo de Mao, la mayor urgencia radicaba en realizar una transformación socioeconómica que permitiera el desarrollo de la población en el menor tiempo posible. Esto se vinculó a la idea de exportar la revolución a través del mundo, que durante la Guerra Fría le permitió acercarse al tercer mundo y proveer importantes recursos a través del discurso confrontacional ideológico y político contra Occidente y el colonialismo. Para la élite política de aquel entonces, este era el medio de realzar el estatus internacional de China, a pesar de que esta misma estrategia lo llevó a involucrarse en conflictos de poder regional característicos del balance de poder entre EE. UU. y la Unión Soviética.

La etapa china de Guerra Fría generó muchas de las bases que le han permitido mayor cercanía y sensibilidad ante las necesidades del actual mundo en desarrollo. Pero esta misma etapa tiene una alta carga ideológica apoyando movimientos revolucionarios de izquierda en el Tercer Mundo, enfrentamientos contra Occidente y el imperialismo occidental, del cual fueron víctimas durante los siglos xix y xx. Todo lo anterior unido a las campañas en apoyo a la descolonización, solidaridad con el mundo en desarrollo, su participación en las guerras de Corea y Vietnam contra el poder militar de EE. UU. y el desarrollo exitoso de su programa nuclear. Esta etapa, más que ninguna, marca los principios para 
la constante búsqueda de poder militar convencional que le permita tener poder persuasivo.

Es importante destacar que la política exterior china depende fundamentalmente de la política interna y responde específicamente a los intereses internos. Esta retórica ha sido utilizada por el Partido Comunista Chino (PCC) desde el mismo momento que inició su causa revolucionaria, con lo cual a través del tiempo ha mantenido legitimidad política al interior de su población, considerándose como el instrumento que devolverá el estatus perdido de China en el mundo.

Sin embargo, a pesar de que se habían dejado las raíces para aumentar su importancia internacional, el mundo bipolar de guerra fría y su poca participación en escenarios multilaterales mantenían a China aislada del mundo. Lo que para la élite parecían los principios de su reconocimiento, le jugaban en contra por la alta carga ideológica de la Guerra Fría, sumado a los procesos internos estructurales y culturales que padecía China, producto del modelo stalinista.

El cambio de métodos en que China buscaba conseguir estatus internacional resulta a partir de 1970 con los primeros acercamientos entre Washington y Pekín, incluyendo el reconocimiento de la República Popular de China por parte de las Naciones Unidas. Estos hechos aumentaron sustancialmente la participación china en escenarios multilaterales, lo que llevó a un cambio en la política exterior confrontacional revolucionaria, que, aunque dependiente de los postulados internos del PCC, había llegado a implementarse por el nuevo programa de reforma y apertura, posterior a la muerte de Mao.

Las diferentes variaciones internas dirigidas a liberalizar la economía surtieron efecto, lo que aumentó el reconocimiento internacional de China por las altas tasas de crecimiento económico que sostuvo durante más de cuatro décadas. Asimismo, su mayor participación en los asuntos internacionales, la llevó a alinear sus políticas internas con una política exterior acorde a sus objetivos de desarrollo económico y los problemas compartidos con el resto del mundo.

Sin embargo, vale destacar que la represión en Tiananmén puso a las élites gubernamentales del país asiático contra las cuerdas. Los retos diplomáticos, sumados a las sanciones impuestas por los diferentes estados e instituciones, le obligaron a aumentar su activismo en los escenarios internacionales, el cual ya venía incrementando regionalmente por vía del multilateralismo. 
Finalizando el siglo xx, en el mundo de posguerra fría, China comenzó seriamente a buscar el mundo multipolar, la democratización de la toma internacional de decisiones, consolidar la unidad del mundo en desarrollo, mejorar la relación con sus vecinos y establecer varias alianzas estratégicas con grandes potencias o bloques de estados. Esto se hizo de la manera más significativa con Rusia para balancear la única superpotencia, EE. UU. (Li, 2012). Sin embargo, el fantasma de Tiananmén contrastaba estos esfuerzos de política exterior. Es importante reconocer que EE. UU. es la superpotencia, y los principios liberales occidentales de protección de los derechos humanos, la democracia y la libertad provienen de los siglos del mandato colonial, pero han sido integrados dentro de los instrumentos internacionales. A través de estos instrumentos internacionales y por medio del mismo multilateralismo que China estaba explotando, debió atravesar una crisis diplomática que iba acompañada por sanciones de diferente tipo desde occidente.

El fantasma represivo se convirtió en el antagonista, frente a una gran potencia que, en política exterior, procura una mayor democratización en la toma de decisiones en las instituciones globales. El discurso pacífico de sus relaciones internacionales, su sensibilidad con las causas para el desarrollo y la creciente imagen que refleja de poder responsable, quedaban eclipsados. Sin embargo, la duda permanece tenue frente al imaginario colectivo, pero en el derecho internacional, en el artículo 38 del Estatuto de la Corte Internacional de Justicia, "la costumbre internacional" sirve como "prueba de una práctica generalmente aceptada como derecho", por lo cual la costumbre es una forma espontánea de creación del derecho, porque surge por la práctica. Al ser este un artículo académico, me suscribiré a las características de las acciones internacionales de China, que como explica el derecho internacional, surgen de una práctica recurrente y generalmente aceptada.

Para Pekín consolidar su estatus internacional en el siglo xxI es mucho más redituable, si se tiene en cuenta que se ha enfocado únicamente en lograr dos puntos básicos: 1. alcanzar reconocimiento dentro de la comunidad internacional y 2. cimentar su legitimidad política externamente. De esta manera, los tomadores de decisiones del país asiático construyeron una política exterior dependiente como la de ningún otro Estado del cooperativismo. Además, su enfoque al multilateralismo le ha ayudado a crear, dentro de su discurso y planteamientos, un sentido de seguridad, comunidad y crecimientos económicos mutuos (gana-gana). 
Comparado con la pax britannica y la pax americana, caracterizadas ambas por su desarrollo belicista, conquistador y colonialista, en las cuales se imponían modelos de gobierno, se irrespetaba la soberanía estableciendo fronteras a discreción y se presionaba militarmente a los estados para apoderarse de sus proyectos estratégicos. China padeció el colonialismo como muchos de los países en desarrollo, por lo cual, las élites pretenden que la transición a superpotencia de China se construya en principios y fundamentos diferentes a los acostumbrados por los anteriores colonizadores y potencias occidentales. Lo anterior demuestra que algunos principios estipulados por Mao, en la primera fase de la política exterior China, siguen vigentes en la actualidad, en la cual el tercer mundo hoy se conoce como mundo en desarrollo. La pax sínica se erige a partir del cooperativismo, el multilateralismo, la asistencia en infraestructura para el desarrollo y el entendimiento mutuo. Un ejemplo de estas características puede identificarse con la "Declaración de conducta de las partes en el mar del sur de China" firmado en Nom Pen, Camboya en el 2002, en la cual se mantuvo el libre tránsito de naves y se fortaleció la comunicación en favor de unas relaciones pacíficas en la región (Asean, China).

China se muestra como una gran potencia, que sigue trascendiendo ante el mundo como una potencia responsable, legítima y sensible a las amenazas experimentadas por terceros países. Un ejemplo de este comportamiento es su destacada cooperación con la Asean y sus vecinos, posterior a la crisis financiera de 1998, la cual golpeó al Este de Asia drásticamente. China, en conjunto con Japón y Corea, fueron los grandes prestamistas para el establecimiento de los bonos swaps, que, con la ayuda de los países del Sudeste Asiático, establecieron el fondo de estabilización conocido como la Iniciativa Chiang-Mai (Asean+3). Esta herramienta dotó de la liquidez suficiente a sus participantes, para hacer frente a futuras crisis financieras y tener margen de acción ante la inmovilidad e ineficiencia demostrada por las instituciones financieras internacionales durante la crisis y los pobres resultados de sus paquetes de ajuste estructural.

\section{China y las características de una gran potencia}

Es importante establecer las características del poder convencional con el que cuenta China actualmente y, además, enriquecer el análisis 
de su tránsito de gran potencia en transición a superpotencia. Para este fin, utilizaré los términos de Buzan (Buzan, 2003), quien en su teoría de los complejos regionales de seguridad, define tres tipos de estado: superpotencias, grandes potencias y potencias regionales. Lo que interesante de esta teoría son los niveles de análisis sobre los que estos actores podían ejercer acción en los ámbitos global o regional. La superpotencia y las grandes potencias tienen penetración en el contexto global y trascienden las fronteras regionales, mientras que las potencias regionales definen los complejos regionales de seguridad, pero no tienen alcance global.

Siendo las superpotencias aquellas que poseen capacidades políticomilitares de primera clase (según lo medido por los estándares del día) y las economías para respaldar dichas capacidades, deben ser capaces de tener y también ejercer un poder militar y político global. Necesitan verse a sí mismas y ser aceptadas por otros en retórica y comportamiento. Las superpotencias deben ser actores activos en los procesos de segurización y desegurización en todas o casi todas las regiones del sistema, ya sea como amenaza, garante, aliado o interventor.

Por otro lado, las grandes potencias poseen menor capacidad. Las grandes potencias no necesariamente tienen que tener grandes capacidades en todos los sectores y no necesitan estar activamente presentes en los procesos de segurización de todas las áreas del sistema internacional. Para que sean consideradas grandes potencias es básico considerar una clave que las distingue de las meramente regionales, esto es, que responden por otros, sobre la base de los cálculos de distribución del poder presente y en el futuro cercano. Por lo general, esto implica que una gran potencia se trata en los cálculos de otras grandes potencias, como si tuviera el potencial económico, militar y político claro para alcanzar el estado de superpotencia en el corto o mediano plazo. Esta clave única es observable en los procesos de política exterior y discursos de otras potencias. Esto significa que la posesión real de atributos materiales y legales es menos crucial para las grandes potencias que para las superpotencias. Las grandes potencias generalmente tendrán niveles adecuados de capacidad, aunque China ha demostrado una capacidad impresionante durante casi un siglo para negociar con las capacidades futuras que aún no ha conseguido.

Los poderes regionales definen la polaridad de cualquier complejo regional de seguridad dado: unipolar, como en el sur de África; bipolar, como en el sur de Asia; multipolar, como en el Medio Oriente, Sudamérica 
y el sudeste de Asia. Sus capacidades son grandes en sus regiones, pero no se registran mucho en un amplio espectro en el contexto global.

Se señalarán a continuación los elementos característicos de las grandes potencias de acuerdo con los neorrealistas, para asimismo hacer un paralelo con la actualidad de China y demostrar su transición de gran potencia a superpotencia, por lo cual se realizarán comparaciones en lo referente al poder convencional, en el aspecto militar y político.

\section{La potencialidad material o fuerza potencial}

a. La riqueza material: la disponibilidad de todos aquellos recursos naturales suficientes para permitir una escasa o nula dependencia exterior en el abastecimiento de estos, dado un determinado grado de evolución máxima del desarrollo socioeconómico del país.

China es el cuarto país más grande del mundo en área total y es el quinto país con mayor número de recursos renovables de agua fresca, con un total de $2.132 \mathrm{Km}^{3}$. Esta misma capacidad hídrica le permitió ser una de las potencias mundiales en energía hidroeléctrica, y cuenta con la presa de las tres gargantas, que produce la mayor cantidad de energía en el mundo con 22.500 Mw. En el 2015, estadísticas del Banco Mundial indicaron que China poseía al menos el 12,7\% de la tierra cultivable en el mundo. De acuerdo a varias iniciativas del gobierno, se ha experimentado un crecimiento en estas tierras cultivables desde el 2015, lo que convierte este en un importante sector de su economía, en el cual se ofrecen cerca de 300 millones de empleos.

En cuanto a la minería, China también se destaca por contar con grandes reservas de carbón, oro, aluminio y plomo. Dentro de este punto vale destacar que en la actualidad, China es el mayor productor mundial de oro: en el 2017 alcanzó una producción de 426 toneladas.

b. Recursos demográficos: se refiere a la existencia de una base demográfica suficiente para permitir la máxima expansión de la capacidad productiva y militar del Estado.

De acuerdo a la Oficina Nacional de Estadística de China, en el 2017, la población total alcanzó 1.390.080 millones de habitantes. Debe remarcarse que la tendencia de crecimiento demográfico se ve debilitada, gracias al impacto de la "política de 
un solo hijo". En concordancia con el documento "Prospectos de la población mundial de las naciones unidas" (2017), se espera que China empiece una reducción sistemática de sus habitantes. Sin embargo, a pesar de reducir la presión en el crecimiento, seguirá contando con una población bastante alta, como para enfrentar los retos de ser una gran potencia.

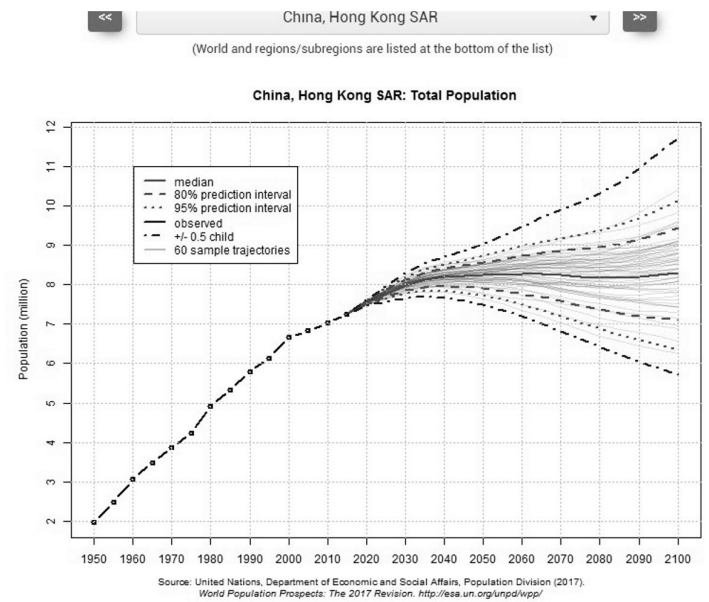

Figura 1. Proyección de la población China.

Fuente: Naciones Unidas (2017).

\section{China's Changing Population}

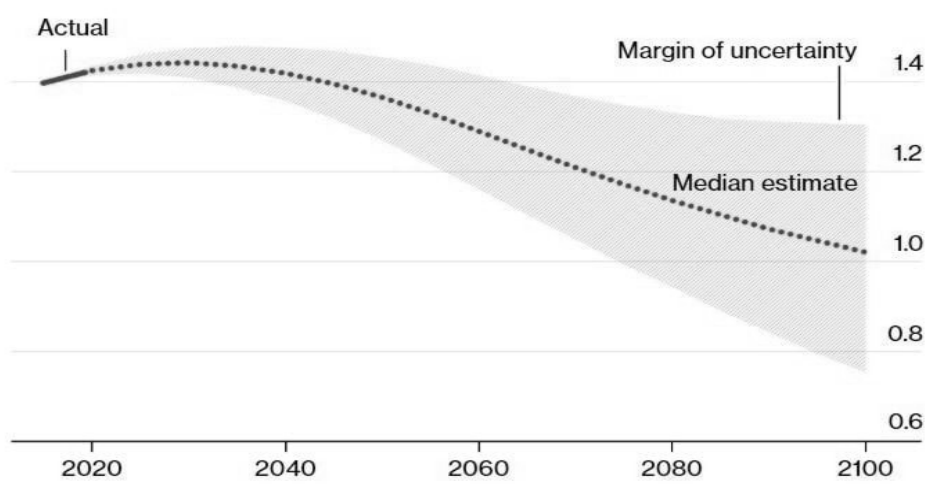

Figura 2. Cambio en la tendencia poblacional china.

Fuente: Naciones Unidas (2017).

c. Desarrollo político-administrativo y tecnológico: el Estado debe gozar de un grado de organización político-administrativa interior para la óptima utilización de los recursos disponibles. 
Además, debe poseer una autosuficiencia tecnológica que le ayude a mantener su propio proceso de expansión económica y militar sin estar sujeto a condicionamientos externos decisivos.

En cuanto al desarrollo político-administrativo, la revolución cultural que duró 10 años, no solamente deterioró la economía, sino que destruyó completamente el sistema de administración pública. Sin embargo, el poder político en China ha demostrado la más efectiva utilización de los recursos disponibles. Como evidencia se cuenta con las reformas realizadas y los resultados obtenidos en su modernización y mejoramiento de procesos económicos, con una nueva iniciativa emergiendo cada cinco años. El enfoque de estas reformas era diferente en su escala, foco y resultado, pero relativamente coherente e incremental en naturaleza. Muchas de estas reformas respondieron a ineficiencias y fallas de los programas anteriores, lo que buscaba un impacto profundo para resolver problemas socioeconómicos (Wang, 2010).

Como resultado de la estrecha relación entre la política partidista y la administración, cualquier reforma es construida como un componente de la reforma política. A diferencia de la práctica sostenida en las democracias occidentales, el Partido Comunista administra el servicio civil directamente: controla el nombramiento de servidores civiles políticos por medio de los comités al interior del Congreso del Pueblo. También mantiene el control de personal administrativo clave y servidores públicos de carrera, basado en la verificación de sus estándares políticos. Aunque "separar el partido del gobierno" ha sido el eslogan utilizado desde 1987, este ideal aún no ha sido materializado. Una explicación radica en que la reforma administrativa está estrechamente vinculada con una reforma económica y con una reforma política (Wang, 2010). Vale destacar que esta centralización de los procesos ofrece un mayor control a partir del ejercicio de la autoridad y la definición de los objetivos plasmados por un poder central que tiene prácticamente el control sobre cada uno de los servidores de la administración.

Respecto de los aspectos tecnológicos, China ha desarrollado la estrategia "hecho en China 2025", la cual fue formulada por el Ministerio de Industria y Tecnologías de la Información. El objetivo fundamental es convertir la industria manufacturera en una mayormente dirigida a la innovación, enfatizando la 
calidad sobre la cantidad y reestructurando la famosa industria manufacturera china de bajo costo.

BRI es una herramienta fundamental para facilitar la exportación de productos de alta gama, manufacturados en China. En este sentido, los decisores chinos consideran que los mercados emergentes serán más proclives a recibir bienes industriales de alta gama, que aquellos países desarrollados. Pekín pretende enfocarse en exportar sus estándares tecnológicos y que estos la lleven a convertirse en una economía basada en la innovación, la investigación y el desarrollo.

d. Capacidad militar disuasoria: también deberá China disponer de armamento que le permita participar en los conflictos bélicos internacionales con garantías de éxito o, en todo caso, poder utilizar este armamento para amenazar de modo creíble al posible adversario. En la actual situación internacional, esto requiere la disponibilidad propia de armas tanto convencionales como nucleares tácticas, pero no necesariamente de armamento nuclear estratégico (por ejemplo, misiles intercontinentales).

Adam Ni, un experto sobre el ejército de China en la Universidad Nacional de Australia, escribió para el interés nacional en marzo del 2018 que Pekín ya ha hecho grandes avances en aviones no tripulados, aviones de combate ocultos, misiles y más. También está haciendo grandes avances en inteligencia artificial y computación cuántica que podrían dar a las fuerzas armadas de Pekín una ventaja sobre la tecnología en manos de las tropas estadounidenses. Pero el verdadero avance, señala la comisión, está en los misiles hipersónicos. En agosto, por ejemplo, China probó uno que podría ser casi imposible de detener para los EE. UU. porque vuela seis veces más rápido que la velocidad del sonido. Asunto que despierta temor, especialmente porque un misil de este tipo podría llevar ojivas nucleares (Ward, 2018).

Sin embargo, cuando se trata de la disuasión nuclear, China sigue siendo un jugador poco relevante, en comparación con los EE. UU. o Rusia. China ha mostrado más interés en desarrollar una ventaja en las nuevas tecnologías, como los misiles hipersónicos, la inteligencia cibernética y la inteligencia artificial, demostrando su liderazgo moral al no tratar de 
igualar las reservas nucleares que quedaron de la Guerra Fría (Champion, 2018).

En la figura 3 se presenta el presupuesto aprobado para el gasto militar en los países que cuentan con mayor gasto en seguridad y defensa. En la figura 4, se comparan las capacidades de China frente a las de EE. UU., teniendo en cuenta que el segundo posee la mayor capacidad disuasiva del planeta.

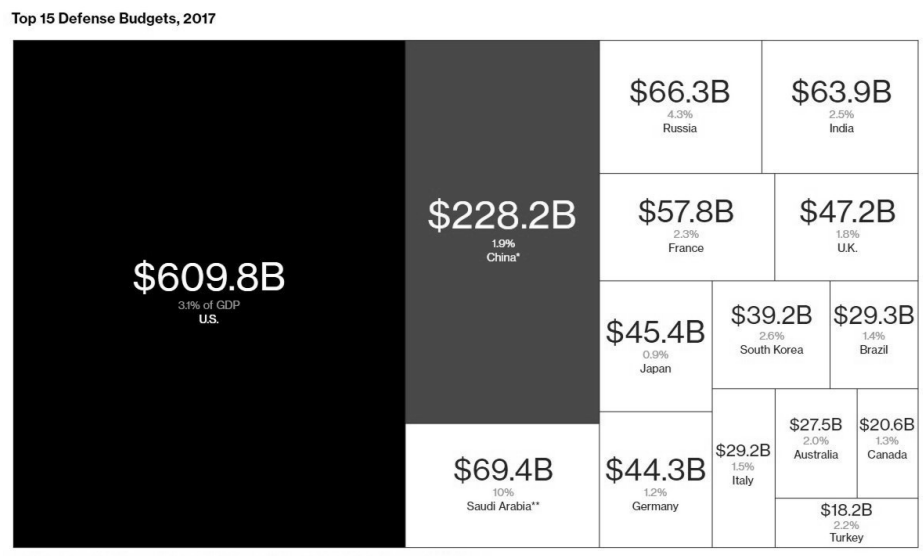

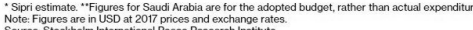

Figura 3. Presupuesto para gasto militar 2017. Nota. * Estimados Sipri ***Para

Arabia Saudita se utiliza el presupuesto aprobado, en vez del gasto actual.

Los datos están en USD a precios y tasas de cambio del 2017.

Fuente: "Does China Have What It Takes to Be a Superpower?" (2018).

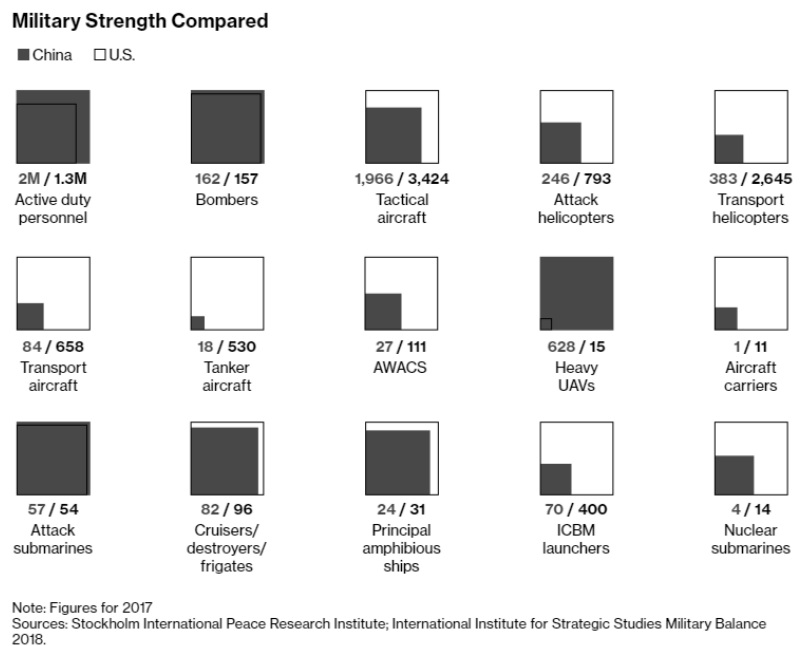

Figura 4. Comparación capacidad militar China frente a EE.UU. (2017). Nota.

Cifras del 2017.

Fuente: "Does China Have What It Takes to Be a Superpower?” (2018). 
Autonomía y control económico significativos internacionalmente:

el Estado debe gozar no solo de la capacidad para satisfacer las demandas económicas interiores de un modo autosuficiente, sino también debe gozar del suficiente poderío económico para crear y mantener un área internacional, en cuyo seno las relaciones económicas (comerciales y financieras) se encuentren nucleadas en torno a la economía de dicho país.

Las raíces del BRI se encuentran en varios desarrollos que hacen plenamente significativo este aspecto. El primero es el surgimiento de China como potencia económica mundial y una economía cada vez más globalizada, como resultado de la absorción por China de las entradas masivas de IED y la experiencia de gestión y la adopción de un modelo de desarrollo orientado a la exportación. En el 2014, China fue el segundo país más grande en términos del PIB, el más grande en el comercio de productos básicos y el tercero más grande en el exterior directo Inversión (OFDI). En los últimos años, China representó alrededor del 25-30\% del incremento anual de crecimiento del PIB mundial. Tan solo tres décadas antes, China era una economía pequeña y semicerrada representando solo el $5 \%$ del PIB mundial, el 1,5\% de las exportaciones mundiales, pero con el $23 \%$ de las exportaciones mundiales. En el 2014 las dos primeras cifras alcanzaron el 13,4\% y el $12,2 \%$ respectivamente. China es centro de manufactura del mundo, que representa aproximadamente el $24 \%$ de la producción mundial en comparación con el $20 \%$ de los EE. UU., y ocupa el primer lugar en la producción de más de 200 bienes fabricados. Además, la ofDi aumentó a más de usD 100 mil millones anuales en los últimos años desde solo varios miles de millones una década antes (Weidong Liu, 2016, p. 7).

e. Una potencia activa o una fuerza actual: entendiendo bajo estas expresiones la movilización efectiva de las capacidades de un Estado para intervenir en el panorama internacional con objetivo de alcanzar los objetivos y fines establecidos por la política exterior de este Estado.

El mantenimiento de la paz es otra forma no coercitiva de proyectar influencia en todo el mundo. De no desempeñar casi ningún papel en las misiones de mantenimiento de la paz de las Naciones Unidas hace 20 años, China es hoy en día el mayor contribuyente de tropas. También es el Estado con el segundo pago más alto, al alcanzar más del $10 \%$ del presupuesto total de la onU. 


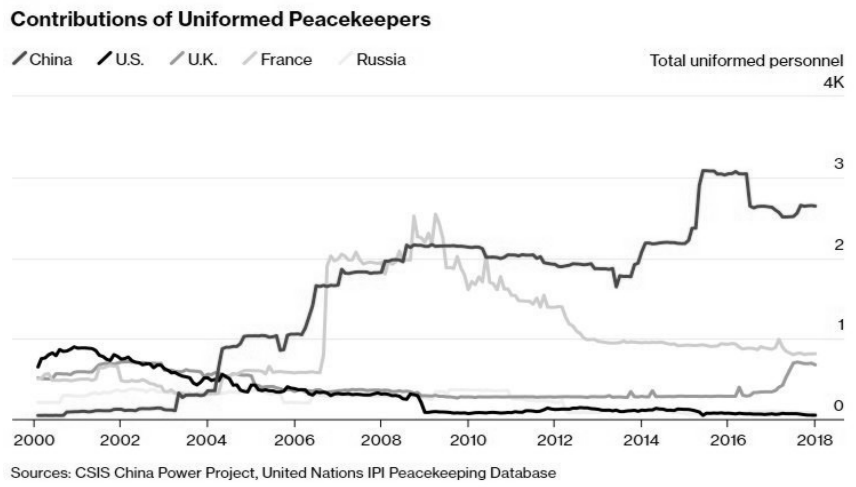

Figura 5. Uniformados enviados a operaciones de mantenimiento de la paz de la ONU. (2018).

Fuente: "Does China Have What It Takes to Be a Superpower?” (2018).

f. La voluntad política, o movilización, constituye el nexo entre la potencialidad material y la potencia activa, ya que para poder transformar una en otra hace falta el concurso de una voluntad política y una capacidad decisional y ejecutiva que permita la utilización adecuada de todos los recursos disponibles para alcanzar y mantener una posición hegemónica y dominante, propia de toda gran potencia, en el panorama internacional. La ausencia de esta voluntad política de convertirse en una gran potencia hace que un Estado, aun disponiendo de unos recursos humanos, materiales y organizativos para lograr una posición hegemónica internacional, nunca llegue a protagonizar una verdadera posición de gran potencia ni alcance a ser reconocido por los demás estados como tal, al carecer de la formulación de una política exterior orientada a lograr semejante objetivo (Calduch, 1991). Esto puede demostrarse a partir de las cifras de la figura 5, con la búsqueda de ser un poder responsable, desde principios de siglo ha comenzado a enviar el mayor número de personal de seguridad y defensa a operaciones de mantenimiento de la paz.

\section{BRI: la globalización incluyente y el modelo chino de desarrollo}

El 7 de septiembre del 2013, el presidente chino Xi Jinping ofreció un discurso en la Universidad Nazarbayev, en Kazajistán. En esta intervención 
expresó: “[...] para forjar lazos económicos cercanos, profundizar la cooperación y expandir el espacio de desarrollo en la región euroasiática, deberíamos tomar un enfoque innovador y conjuntamente construir un cinturón económico a través de la Ruta de la Seda”. Dentro de su discurso remarcó que los más de 2.000 años de historia y de contacto han demostrado que las naciones con diferentes orígenes raciales, religiosos y culturales, bien pueden compartir la paz y el desarrollo común, mientras se adhieran a principios, como la unidad, igualdad, cooperación, adaptación, confianza mutua y beneficio mutuo. Siguiendo el mismo estilo de construcción discursiva frente al parlamento de Indonesia durante la misma gira por Asia Central y el Sudeste Asiático en el 2013, Xi presentó el segundo pilar de su plan: propuso construir conjuntamente con las naciones de la Asean la Ruta Marítima de la Seda del siglo xxi.

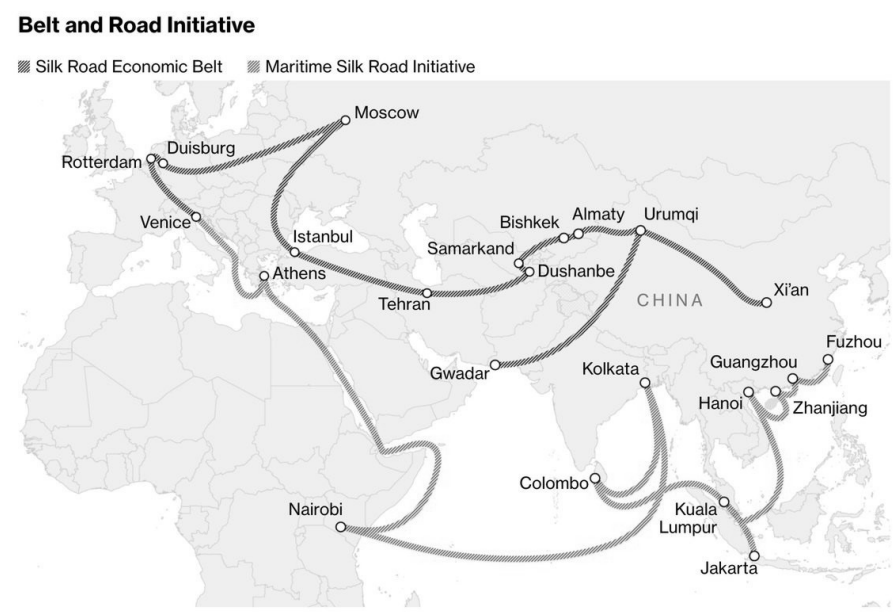

Sources: Belt and Road Portal, China's National Development and Reform Commission

Figura 6. Iniciativa del Cinturón y la Ruta de la Seda.

Fuente: "Does China Have What It Takes to Be a Superpower?" (2018).

El One Belt One Road (овоR) fue completamente incorporado a la Estrategia Nacional China para el Desarrollo Económico; esta decisión fue acogida al interior de la Conferencia Central de Trabajo Económico llevada a cabo en el 2014. Esta conferencia es un evento anual, en el cual se contó con la presencia del presidente Xi Jinping, el premier Li Keqiang y el vicepremier Liu He como las figuras más relevantes. También estuvieron acompañados de oficiales de regulación financiera, planeadores económicos, gobernadores, dirigentes del Congreso Nacional Popular, la Corte Suprema, la Procuraduría Suprema Popular, empresas estatales, 
instituciones financieras y el Ejército Popular de Liberación. En teoría debían asistir 204 miembros del Comité Central del Partido Comunista y 172 miembros alternativos junto con una lista de delegados invitados ("5 things to know about China’s Central Economic Work Conference as policymakers plan US trade war fightback”, 2018).

En consecuencia, Pekín anunció tres planes de desarrollo regionales, y OBOR era uno de estos. Este proyecto le sirvió a China para conectar sus territorios occidentales con los centros económicos más cercanos, lo que integró los mercados de los países vecinos, para que, por medio del comercio, se logre mejorar el nivel de ingresos percibidos por las poblaciones de la zona y aumenten el empleo a través de los proyectos de infraestructura y el incremento de los intercambios comerciales, en una economía que orientó su desarrollo en las exportaciones. Por esta razón, la agencia del gobierno que coordina la iniciativa es la Comisión de Desarrollo Nacional y Reforma, junto con el apoyo técnico del Ministerio de Relaciones Exteriores y el Ministerio de Comercio.

Los proyectos nacionales relacionados con la Ruta de la Seda serán la prioridad para el Gobierno chino, teniendo en cuenta que puede realizar los ajustes que sean necesarios de forma efectiva, debido esto a que estos proyectos están sometidos a sus propios estándares y ordenamiento jurídico; a diferencia de aquellos componentes que se tienen planeados en el exterior, en los cuales existe cierta dependencia a las instituciones económicas, sociales y políticas de los socios del BRI.

La Ruta de la Seda contiene una fuerte construcción discursiva, la cual, para Weidong Liu (2016), resulta ser una metáfora en sí misma. Tanto las instituciones como los círculos académicos defienden y construyen tanto el espíritu como los principios de una nueva era para la cooperación internacional, proyecto que representa un viraje frente al modelo de desarrollo apoyado en el consenso de Washington. BRI es la herramienta por medio de la cual se genera un lenguaje, en el cual se enmarcarán los principios y fundamentos bajo los cuales la segunda economía con el mayor PIB en el ámbito mundial, construyó su propio destino manifiesto basado en la globalización incluyente.

Durante el 2007, el presidente del Banco Mundial, Robert B. Zoellick, se refirió por primera vez la "globalización incluyente". De esta manera expuso que esta “[e]s la visión del Grupo del Banco Mundial contribuir a una globalización incluyente y sostenible: superar la pobreza, mejorar el crecimiento cuidando el medio ambiente y crear oportunidades y esperanzas individuales" (Zoellick, 2007). 
El espíritu de esta expresión sirvió para la formulación del discurso necesario para estructurar "el Cinturón y la Ruta de la Seda”. Este concepto es una crítica inmanente de la globalización neoliberal de los últimos treinta o cuarenta años; la globalización incluyente incorpora similitudes y diferencias fundamentales con la primera. La globalización incluyente no implica la desglobalización o revertir la globalización, sino más bien un desarrollo y una reforma fundamental de la globalización. De esta manera, la globalización inclusiva y la neoliberal van en la misma dirección. La diferencia fundamental entre los dos consiste en que la globalización incluyente está diseñada, ante todo, para mejorar los medios de vida de las personas, en lugar de servir únicamente a los intereses del capital. Segundo, los mercados de capital requieren una reforma para dirigir la inversión a infraestructura y actividades productivas. En tercer lugar, a diferencia de un camino neoliberal impuesto desde arriba, los países deberían elegir caminos de desarrollo que se adapten a sus propias condiciones nacionales. Cuarto, la participación debe estar abierta a todos y generar beneficios mutuos. Finalmente, la diversidad cultural (y el medio ambiente) debe ser protegida.

Desde la década de 1980, con el predominio creciente de las ideologías neoliberales que abogaban por la liberalización del comercio, la inversión y la retirada del Estado de las actividades de gestión económica nacional (medidas del Consenso de Washington). La agenda de la reforma neoliberal redistribuyó las funciones y el poder de los actores públicos a los privados, redujo la capacidad de los estados territoriales modernos y devolvió las funciones estatales residuales a las administraciones subordinadas.

Sin embargo, los países que siguieron las medidas nacionales del consenso de Washington (América Latina, África Subsahariana y la anteriormente comunista Europa del Este) no tuvieron un buen desempeño. China, a la inversa, aprovechó la reubicación de industrias para promover un rápido crecimiento económico. Si bien el rápido crecimiento en China experimentó un aumento en la desigualdad (que es de esperar como un país inicialmente industrializado y urbanizado), el crecimiento elevó a todos, en el sentido de que los ingresos de la mayoría de los sectores de la sociedad aumentaron, aunque a diferentes tasas.

La recuperación de China desempeñó, además, un papel importante en la reducción de la pobreza (crecimiento inclusivo) en marcado contraste con gran parte del resto del mundo donde la globalización neoliberal vio el aumento de la pobreza. En 1981-2005, un modelo de desarrollo 
inclusivo sacó a 627,4 millones de chinos de la pobreza, que obtenían un ingreso diario de USD 1,25. En el mundo, excluyendo a China, el número de pobres aumentó en 107,9 millones (Ravallion, 2010). La razón de estas diferencias era que "las virtudes de la mano invisible del mercado" estaban equilibradas con "las virtudes del buen gobierno" (Mahbubani, 2008).

El éxito económico de China fue el resultado de la capacidad del gobierno chino para adoptar un modelo efectivo de crecimiento orientado a la exportación. Esta estrategia involucró varios elementos. Primero, la nueva China desarrolló un fuerte sentido de identidad nacional y un estado fuerte capaz de entregar bienes colectivos (salud, educación, ley y orden, infraestructura y una política industrial). En segundo lugar, China pudo ejercer control sobre su cuenta de capital, lo que permitió al Banco Central acumular reservas y al Gobierno a crear un fondo de riqueza soberana. La acumulación de reservas ejerció una presión a la baja sobre el tipo de cambio de China, reforzando la competitividad de las exportaciones, la rentabilidad y la inversión, y limitando el consumo y las importaciones, aunque las reservas negativas se invirtieron en valores gubernamentales seguros a corto plazo con tasas de interés bajas. En tercer lugar, China movilizó el ahorro interno, junto con la inversión extranjera directa, y transformó esto en inversiones en agricultura, infraestructura y capacidad industrial doméstica intensiva en capital, equipos y bienes de consumo. La acumulación de reservas también significó que China estaba generando más ahorros de los que gastaba en la inversión doméstica proporcionando crédito a los países desarrollados que importaban sus exportaciones. Cuarto, China desarrolló una política industrial en la que la protección efectiva se combinó con la promoción de exportaciones y se alentó la sustitución de importaciones, pero la orientación de las exportaciones disciplinó y alentó a las industrias nacionales a ser competitivas en el contexto internacional. La lección de esta experiencia es, por lo tanto, que la capacidad de aprovechar la globalización depende de la capacidad del Estado, que en realidad se redujo en los países que adoptaron las agendas de reforma neoliberales nacionales.

En este sentido, es importante recoger que los lineamientos de China para alcanzar el liderazgo mundial se apartan de toda construcción discursiva de grandes potencias anteriores, debido a que, para el país del Este de Asia, el camino hacia el desarrollo tiene como pieza clave las diferentes naciones en desarrollo, las cuales también ayudarán a superar retos nacionales chinos que tienen repercusión global, como lo es el exceso de capacidad. Lo anterior puede observarse en el párrafo a continuación, 
en el cual se demuestra la construcción discursiva, los fundamentos y principios de este ambicioso proyecto que marcará el futuro de la República Popular y su estatus en el mundo:
En el siglo xxi, una nueva era marcada por el tema de la paz, desarrollo, cooperación y beneficio mutuo, sean lo más importante para todos los participantes y que permita portar y extender el espíritu de la Ruta de la Seda para enfrentar la débil recuperación de la economía global y las complejas situaciones internacionales y regionales (Comisión de Desarrollo Nacional y Reforma de la República Popular, 2015).

De manera muy consecuente con la búsqueda de la paz, China ofrece reducir las brechas y conflictos entre Este y Oeste. Por esto, plantea, a través de estos principios, evitar cualquier tipo de confrontación económica, política y militar con cualquiera de sus competidores. Así, China plantea lo siguiente para hacer plausible la resolución pacífica de controversias de tipo global que parecen irreconciliables:

\begin{abstract}
Por miles de años, el espíritu de la Ruta de la Seda, "paz y cooperación, apertura e inclusión, aprendizaje mutuo y beneficio mutuo", ha pasado de generación a generación, promoviendo el progreso de la civilización humana y contribuyendo enormemente a la prosperidad y el desarrollo de los países que le conforman. Simboliza la comunicación y cooperación entre el Este y el Oeste, su espíritu es patrimonio histórico y cultural compartido con todos los países del mundo (Xinhua, 2017).
\end{abstract}

BRI es una iniciativa que, de acuerdo a los discursos realizados por Xi Jinping, es abierta a todas las regiones del mundo y busca promover la cooperación intergubernamental, construir intercambios intergubernamentales de política macro y multinivel, junto con mecanismos de comunicación para expandir intereses compartidos, fortalecer la confianza política mutua y alcanzar nuevos consensos de cooperación (Comisión de Desarrollo Nacional y Reforma de la República Popular, 2015).

Sin embargo, los documentos emitidos priorizan la conexiones entre China y los países a lo largo de la antigua Ruta de la Seda y propone construir seis corredores terrestres de transporte: un nuevo puente terrestre euroasiático y China-Mongolia-Rusia, China-Asia Central- 
Asia Occidental, China-Pakistán, Bangladesh-China-India-Myanmar, y los corredores económicos entre China-Indochina, que profundizarán la gran evolución histórica de las relaciones sostenidas con la Asean en las últimas décadas.

\section{Las fuerzas del BRI conllevarán a la transformación de los complejos de seguridad en Asia}

Para poder definir el papel del BRI en la transición a superpotencia, me gustaría acudir a las cuatro variables de un complejo regional de seguridad (Buzan, 2003): 1. límite, que diferencia al complejo regional de seguridad de sus vecinos; 2. estructura anárquica, lo que significa que el RSC debe estar compuesto por dos o más unidades autónomas; 3. polaridad, que cubre la distribución del poder entre las unidades, y 4. construcción social, que cubre los patrones de amistad y enemistad entre las unidades.

Desde su configuración en cualquier instantánea dada en el tiempo, hay tres posibles evoluciones abiertas a un RSC. Primero, hay un mantenimiento del statu quo, lo que significa que no hay cambios significativos en su estructura esencial. Segundo, existe una transformación interna, lo que quiere decir que los cambios en la estructura esencial ocurren dentro del contexto de su límite exterior existente. Esto puede implicar cambios en la estructura anárquica (debido a la integración regional); en la polaridad (causados por la desintegración, fusión, conquista, tasas de crecimiento diferencial o similares), o en los patrones dominantes de amistad/enemistad (debido a cambios ideológicos, cansancio de la guerra, cambios de liderazgo, etc.). Por último, se da una transformación externa, lo que significa que el límite externo se expande o contrae, cambiando la membresía del Rsc y, probablemente, transformando su estructura esencial de otras maneras.

Teniendo en cuenta las evoluciones abiertas de un Complejo Regional de Seguridad, el BRI permitiría, por un lado, una transformación interna y, por el otro, una transformación externa. El BRI tiene la capacidad de generar una transformación interna del Complejo Regional de Seguridad del Este de Asia; esto se debe a que se generarían cambios radicales en la estructura anárquica dada por la integración que permitirán los ferrocarriles de alta velocidad en el Sudeste Asiático, sumado a los puertos marítimos proyectados para el mejoramiento del comercio. El crecimiento 


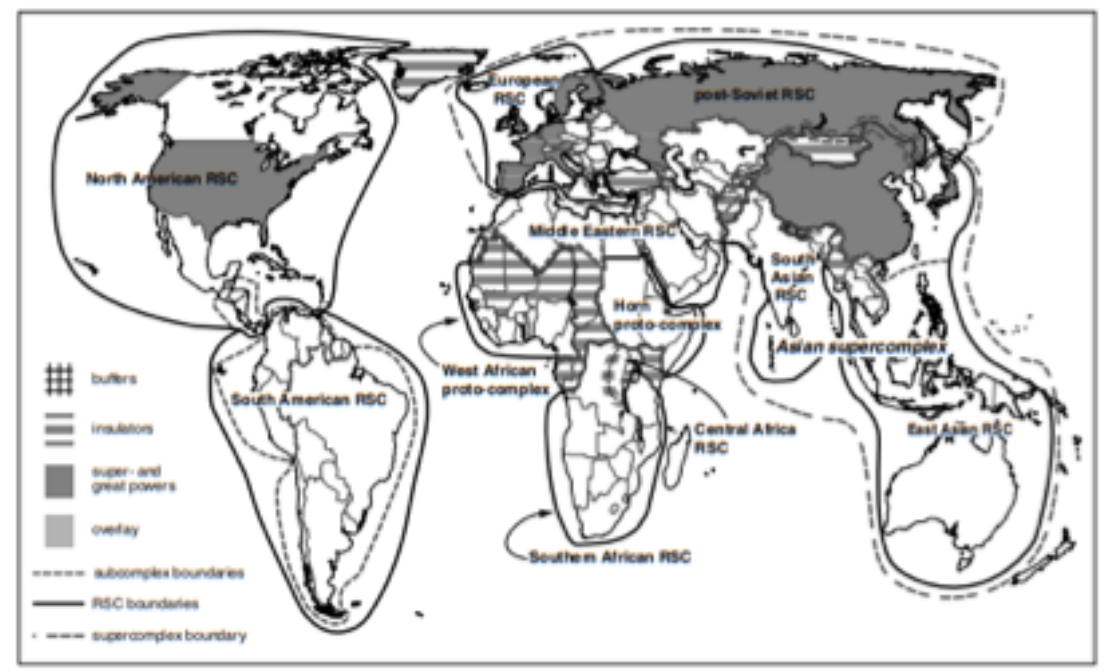

Figura 7. Patrones de seguridad regional en la posguerra fría.

Fuente: Buzan (2003).

en el PIB de China, que se prevé será la mayor economía del mundo para el 2030, generará cambios en la polaridad y transformará las relaciones de amistad o enemistad entre los miembros.

\section{LA RUTA DE LA SEDA EN EL SIGLO XXI}

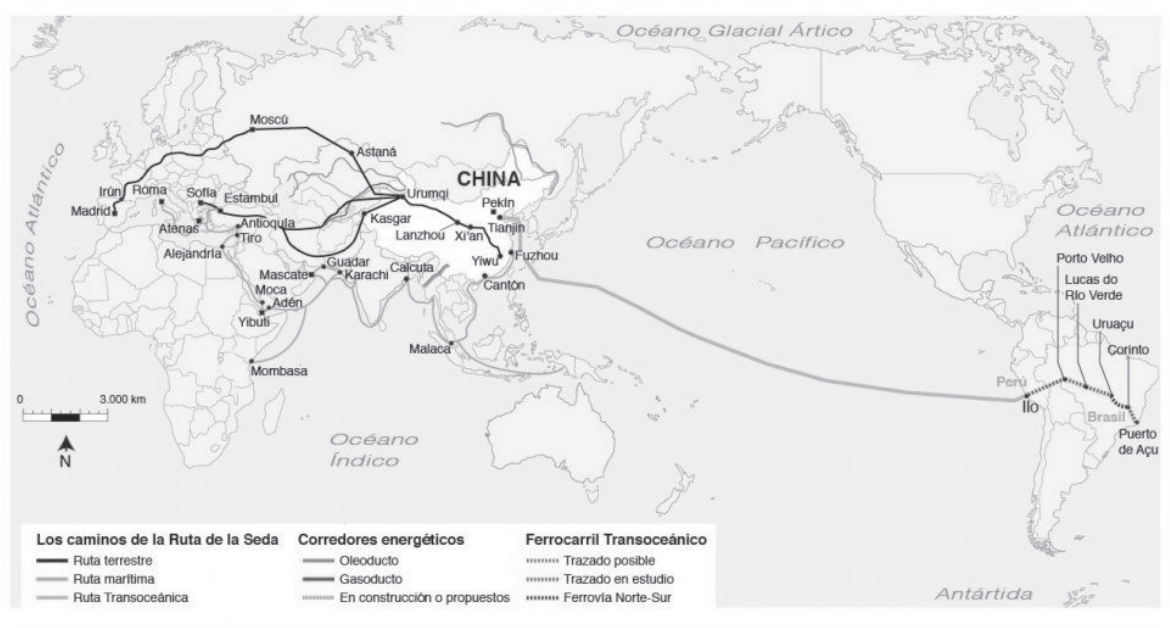

Figura 8. Proyectos de la Ruta de la Seda del siglo Xxi.

Fuente: Higueras (2015). 
Es importante destacar la perspectiva de la teoría de la transición de poder, la cual contempla la política como una jerarquía de naciones con diversos grados de cooperación y competencia. Además, la teoría considera que la política mundial está integrada horizontal y verticalmente. La imagen estática de la estructura y las reglas se complementa con factores dinámicos que demuestran cómo y por qué se produce el cambio en el sistema internacional.

La transición de poder se centra en las tasas de crecimiento diferencial y su efecto en la alteración del poder relativo entre las naciones, lo que da como resultado nuevas relaciones entre las naciones o grupos en competencia, y la formación de nuevas entidades políticas y económicas. Un subproducto del crecimiento diferencial es el alto potencial de conflicto cuando un rival y una nación dominante alcanzan la etapa de equivalencia relativa de poder, y específicamente cuando el candidato no está satisfecho con el statu quo (Kugler, 2011; Organski, 1968).

De acuerdo con lo anterior, el BRI puede percibirse como el proyecto que demuestra la insatisfacción china con el statu quo, además de que representa la fuerza interna y externa de cambio dentro de la estructura de los complejos regionales de seguridad. Como puede observarse en la figura 7, Buzan muestra cómo están organizadas las dinámicas regionales de seguridad. No obstante, al realizar detenidamente un paralelo con la figura 8 , la cual muestra los proyectos clave que pretende llevar a cabo la élite china alrededor del mundo por el BRI, se puede ver que los intercambios culturales y económicos pueden generar un proceso de desegurización entre las fronteras de los complejos de seguridad del Este de Asia, Sur de Asia y el postsoviético.

Otro interesante resultado que ofrece la comparación de ambos mapas es la relegación de América Latina a la periferia, realizando un análisis netamente físico y geográfico de lo que representa la Ruta Económica de la Seda. De la misma forma, esto también significa que se mantendría el statu quo dentro del Complejo de Seguridad de Sudamérica y Norte América.

\section{Conclusiones}

China ha sido fiel a la construcción de identidad nacional, la cual le ha servido para dirigir sus esfuerzos a aumentar su estatus internacional. El Partido Comunista Chino ha utilizado la gloria pasada como un motor de 
orgullo nacional, el cual va a recobrar comprometiéndose a volver a ser el país más poderoso del mundo.

Se puede concluir que el estatus internacional, en la retórica china, mantiene una relación directa con lo que en relaciones internacionales se conoce como poder convencional (hard power), el cual está dirigido a conseguir la mayor innovación y poderío militar posibles. El deseo de China de alcanzar el estatus de superpotencia la lleva a rebatir el statu quo planteado por el poderío de EE. UU. como la única superpotencia. En este sentido BRI es un instrumento a largo plazo para que China tenga una influencia a escala global en los procesos económicos de integración, segurización y desegurización.

China es actualmente una gran potencia; ha propuesto una construcción discursiva para que el BRI sea la herramienta fundamental de reconocimiento global sobre su liderazgo e influencia en las diferentes naciones, utilizando la globalización incluyente y el compromiso que tiene con el mundo en desarrollo. A pesar del fracaso de su primer enfoque de política exterior revolucionaria, los rasgos fundamentales de este direccionamiento siguen plasmados al notarse la sensibilidad China con las causas del desarrollo y la búsqueda del país asiático en financiar proyectos de infraestructura clave para países que no pueden tener la riqueza material para llevarlos a cabo.

Lo anterior también demuestra que China está generando una nueva jerarquía: evidente o no, la generación de deuda para los países beneficiarios ocasiona una nueva jerarquía financiera. Esta nueva jerarquía será un sistema financiero paralelo a los institucionalizados por el Consenso de Washington. La generación de estos flujos de capital puede generar la reorganización del sistema económico internacional, con dos sistemas paralelos basados en modelos de desarrollo diferentes.

\section{Referencias}

5 things to know about China's Central Economic Work Conference as policymakers plan U. S. trade war fightback. (2018, 18 de diciembre). South China Morning Post. Recuperado de https://www.scmp.com/economy/chinaeconomy/article/2178498/5-things-know-about-chinas-central-economicwork-conference

Buzan, B. a. (2003). Regions and Powers: The Structure of International Security. London: Cambridge University Press. doi: https://doi.org/10.1017/ CBO9780511491252 
Cai, P. (2017). Understanding China's Belt and Road Initiative. Analysis - Lowy Institute for International Policy, 1-26. Recuperado de https://think-asia.org/ bitstream/handle/11540/6810/Understanding_Chinas_Belt_and_Road_ Initiative_WEB_1.pdf?sequence=1

Calduch. (1991). Relaciones Internacionales. Recuperado de https://www.ucm.es/ data/cont/media/www/pag-55159/liblcap7.pdf

Champion, L. (2018, 30 de agosto). Does China Have What It Takes to Be a Superpower? Bloomberg. Recuperado de https://www.bloomberg.com/ graphics/2018-china-superpower/

China, Comisión de Desarrollo Nacional y Reforma de la República Popular. (2015). Vision and Actions on Jointly Building Silk Road Economic Belt and 21st-Century Maritime Silk Road. Recuperado de http://en.ndrc.gov.cn/ newsrelease/201503/t20150330_669367.html

Does China Have What It Takes to Be a Superpower? (2018, 30 de agosto). Bloomberg. Recuperado de https://www.bloomberg.com/graphics/2018-chinasuperpower/

Higueras, G. (2015). La Ruta de la Seda del siglo xxi. Estudios de Política Exterior, 2015(167).

Kugler, J. (2011). Power Transitions. International Interactions. Special Issue, 38(5).

Li, M. (2012). International Status: China's Pursuit of a Comprehensive Superpower

Status: The Ashgate Research Companion to Chinese Foreign Policy Routledge.

London: Routledge.

LIU Weidong, M. D. (2018). A discursive construction of the Belt and Road Initiative: From neo-liberal to inclusive globalization. Journal of Geographical Sciences, 28, 1.199-1.214. doi: https://doi.org/10.1007/s11442-018-1520-y

Llagostera, E. (2004). La seda china y la Ruta de la Seda. Boletín de la Asociación Española de Orientalistas, 40, 243-265.

Mahbubani, K. (2009). The new Asian hemisphere: The irresistible shift of global power to the East. New York: PublicAffairs.

Naciones Unidas. (2017). World Population Prospects. The 2017 Revision. Recuperado de https://population.un.org/wpp/Graphs/Probabilistic/POP/TOT/

Organski, A. F. (1968). World Politics. $2 d$ ed. . New York:: Knopf.

Ravallion, M. (2010). Poverty lines across the world. The World Bank.

Wang, Q. (2010). Administrative Reform in China: Past, Present, and Future.

Southeast Review of Asian Studies, 100-119. Recuperado de https://www. academia.edu/16554991/Administrative_Reform_in_China_Past_Present_ and_Future

Ward, A. (2018). China's military power could match America's by 2050. VOX. Recuperado de https://www.vox.com/world/2018/11/14/18091800/chinamilitary-power-congress-commission-report-2050

Liu, W., \& Dunford, M. (2016). Inclusive globalization: Unpacking China's belt and road initiative. Area Development and Policy, 1(3), 323-340.

Xinhua. (2017, 14 de mayo). Full text of President Xi's speech at opening of Belt and Road forum. Recuperado de Xinhuanet: http://www.xinhuanet.com/english/201705/14/c_136282982.htm 
Zoellick, R. (2007, octubre 10). Una globalización incluyente y sostenible.

Banco Mundial. Recuperado de https://www.bancomundial.org/es/news/ speech/2007/10/10/an-inclusive-sustainable-globalization 